\title{
Identification of common differentially-expressed miRNAs in ovarian cancer cells and their exosomes compared with normal ovarian surface epithelial cell cells
}

\author{
SHITAO ZHANG ${ }^{1}$, XIAOPING ZHANG ${ }^{2}$, XUEQI FU ${ }^{1}$, WANNAN LI ${ }^{1}$, SHU XING $^{1}$ and YILING YANG ${ }^{2}$ \\ ${ }^{1}$ Edmond H. Fischer Signal Transduction Laboratory, School of Life Sciences, Jilin University, Changchun, Jilin 130012; \\ ${ }^{2}$ Department of Obstetrics and Gynecology, China-Japan Union Hospital of Jilin University, \\ Changchun, Jilin 130033, P.R. China
}

Received October 21, 2017; Accepted April 23, 2018

DOI: $10.3892 / \mathrm{ol} .2018 .8954$

\begin{abstract}
The aim of the present study was to identify common microRNAs (miRNAs) in ovarian cancer (OC) cells and their exosomes using microarray data (accession number GSE76449) available from the Gene Expression Omnibus database, including exosomal samples from 3 OC cell lines, 1 normal ovarian surface epithelial cell line and their original cell samples. Differentially-expressed miRNAs (DE-miRNAs) were identified using the Linear Models for Microarray data method, and mRNA targets of DE-miRNAs were predicted using the miRWalk2 database. The potential functions of the target genes of the DE-miRNAs were analyzed using the Database for Annotation, Visualization and Integrated Discovery tool. The association between crucial miRNAs and target genes, and their clinical associations, were validated using The Cancer Genome Atlas data. As a result, 12 upregulated and 12 downregulated DE-miRNAs were shared by the 3 OC cell lines compared with normal controls in the exosomal samples, while 5 upregulated and 65 downregulated DE-miRNAs were shared between the original cells. Among them, 9 downregulated DE-miRNAs were shared between exosomal and original cells. The target genes of 4 common DE-miRNAs between exosomal and original cells (miR-127-3p, miR-339-5p, miR-409-3p and miR-654-3p) were predicted. Functional enrichment analysis indicated that these target genes may be involved in the Wnt signaling pathway (miR-409-3p-CTBP1 and miR-339-5p-CHD8) and Proteoglycans in cancer (miR-127-3p-PPP1CA). The negative associations between these 3 miRNAs and target genes were confirmed by a Pearson's correlation analysis. miR-127 was
\end{abstract}

Correspondence to: Dr Yiling Yang, Department of Obstetrics and Gynecology, China-Japan Union Hospital of Jilin University, 126 Xiantai Street, Changchun, Jilin 130033, P.R. China

E-mail: 1129155883@qq.com

Key words: ovarian cancer, exosomes, microRNAs, Wnt signaling pathway, proteoglycans in cancer negatively associated with tumor grade. In conclusion, our results describe a set of miRNAs involved in OC development, in exosomal and non-exosomal manners, by regulating their target genes. They may be potential targets for treatment of OC.

\section{Introduction}

Ovarian cancer (OC) is the most common gynecological malignancy in China, with an estimated 52,100 incident cases diagnosed and $>22,500$ mortalities in China in 2015 (1). Despite advances in its treatment, including surgery, chemotherapy and radiotherapy, survival rates remain poor for the majority of patients, with a 5-year survival rate of $\sim 30 \%$ (2). Therefore, it is essential to develop novel therapeutic strategies.

MicroRNAs (miRNAs) are small non-coding RNAs measuring 19-22 nucleotides in length that regulate gene expression by binding to complementary sequences in the 3 'untranslated region (UTR) of genes, leading to either the inhibition of translation or degradation of the gene transcript (3). Increasing evidence has suggested that miRNAs are involved in OC development and progression. For example, Yang et al (4) demonstrated that miR-23a was upregulated in OC cells, and that it promoted the proliferation, migration and invasion, but repressed apoptosis, of OC cells through the downregulation of the expression of the tumor suppressor gene Suppression of tumorigenicity 7 like, and activation of the Wnt signaling pathways. Shen et al (5) demonstrated that miR-26a was overexpressed in human OC specimens: Ectopic expression of miR-26a increased OC cell proliferation and clonal formation through the inhibition of estrogen receptor (ER)- $\alpha$, which was also confirmed in nude mice. Dong et al (6) demonstrated that $\mathrm{OC}$ tissues exhibited significantly decreased levels of miR-137 and miR-34a when compared with adjacent normal tissues, resulting in a trend towards poorer survival. Additionally, using luciferase assays, miR-137 and miR-34a were demonstrated to inhibit epithelial-to-mesenchymal transition and cell invasion by acting as direct suppressors of zinc finger protein SNAI1 in OC cells (6). Therefore, miRNAs may be potential targets in OC treatment. 
In addition to being present intracellularly, miRNAs may also be secreted extracellularly through membrane-bound vesicles, including exosomes. It has been suggested that exosomal miRNAs may transfer phenotypic traits from the cancer cells of origin into surrounding normal cells, and therefore facilitate tumorigenesis and progression (7). For example, Baroni et al (8) observed that the transfer of breast cancer-secreted miR-9 to normal fibroblasts via exosomes increased the migration and invasion capabilities of recipient normal fibroblasts, contributing to the formation of cancer-associated fibroblasts. Treatment with exosomes derived from metastatic breast cancer MDA-MB-231 cells, including the highly-expressed miR-10b when compared with non-metastatic breast cancer MCF-7 cells or non-malignant breast HMLE or MCF-10A cells, was also observed to induce the invasive ability of non-malignant mammary epithelial cells (9). Therefore, targeting miRNAs in cancer cells and their exosomes may represent a more effective approach for OC treatment.

At present, miRNAs of OC in exosomes have rarely been investigated (10). Ying et al (11) proposed that OC-derived exosomes may release miR-222-3p into macrophages and induce polarization of the M2 phenotype, a tumor-associated macrophage-like phenotype, via inhibiting the suppressor of cytokine signalling-3/signal transducer and activator of transcription-3 pathway, which then promoted the growth and metastasis of OC. Using the microarray data, Kanlikilicer et al (12) compared the miRNAs profiles of OC cells with their exosomes, and indicated that miR-6126 was released from OC cells via exosomes. miR-6126 also significantly decreased the tube-forming, invasive and migratory abilities of OC cells in vitro and inhibited tumor growth (with smaller tumor size and lower weight), cell number (fewer numbers of Ki67-positive cells) and microvessel density (fewer CD31-positive cells) in vivo by inhibiting integrin- $\beta 1$ (12). However, these studies did not investigate the common miRNAs in OC cells or their exosomes compared with normal cells, which was the aim of the present study. The present study preliminarily identified that miR-127-3p, miR-339-5p, miR-409-3p and miR-654-3p were commonly differentially expressed in OC cells and their exosomes and therefore may be underlying important targets for the treatment of OC (13).

\section{Materials and methods}

Data collection. The Affymetrix miRNA microarray data (accession number GSE76449) were collected from the Gene Expression Omnibus (GEO) database (http://www.ncbi. nlm.nih.gov/geo/) (12), in which exosomal samples isolated from 6 human epithelial OC [SKOV3_ip1; A2780_PAR, HEYA8, SKOV3_TR (Taxol-resistant), A2780_CP20 (cisplatin-resistant) and HEYA8_MDR (multidrug-resistant)] cell lines, 1 normal ovarian surface epithelial HIO180 cell line and their original cell samples were downloaded. Each sample had 2 biological repeats, resulting in a total of 28 samples. To simplify the present study, and to exclude the effects of chemotherapy drugs including paclitaxel and cisplatin which were been used in the study of Kanlikilicer et al (12), only the exosomal and original cell samples in the chemo-sensitive
OC cells (SKOV3_ip1, A2780_PAR and HEYA8) and normal cells $(n=16)$ were included in the present study to reveal the mechanism and underlying treatment targets for OC.

Data normalization and identification of differentiallyexpressed miRNAs (DE-miRNAs). The raw data (CEL files) downloaded from the Affymetrix Multispecies miRNA-4 Array platform GPL19117 were preprocessed, including background correction $\log 2$ transformation and quantile normalization, using the Robust Multiarray Average function available in the Bioconductorpackage(version 1.34.2; http://www. bioconductor.org/packages/release/bioc/html/limma.html). The DE-miRNAs between cancer and normal cells were identified using the Linear Models for Microarray data method (14) in the Bioconductor R package (version 3.32.5; http://www.bioconductor.org/packages/release/bioc/html/limma.html). miRNAs with $\mathrm{P}<0.05$ and $\mid \log$ fold change $(\mathrm{FC}) \mid>1$ were considered differentially expressed. To visualize the shared DE-miRNAs between different cell types and between exosomes and their cells of origin, a Venn diagram was generated using a web-based tool accessed September 2017 (http://bioinformatics.psb.ugent. be/webtools/Venn/).

Target genes prediction and functional enrichment analysis. mRNA targets of the DE-miRNAs were predicted using the miRWalk2 database (http://zmf.umm. uni-heidelberg.de/apps/zmf/mirwalk2/), which contains 12 bioinformatic algorithms: Microt4; miRWalk; mirbridge; miRanda; miRDB; miRMap; Pictar2; PITA; miRNAMap; RNAhybrid; RNA22; and Targetscan. Targets were selected when they overlapped in at least 4 of 12 databases. Then, the miRNA-target gene interaction network was constructed and visualized using Cytoscape software (version 2.8; www. cytoscape.org/) (15).

The Kyoto Encyclopedia of Genes and Genomes (KEGG) pathways for these target genes were analyzed using the Database for Annotation, Visualization and Integrated Discovery (DAVID) online tool (version 6.8; http://david abcc. ncifcrf.gov) (16). $\mathrm{P}<0.05$ was set as the cut-off value.

Validation of the association between crucial miRNAs, target genes and clinical characteristics. The miRNASeq data of OC (Level 3) was obtained from The Cancer Genome Atlas (TCGA; https://tcga-data.nci.nih.gov/; accessed January 2018) to confirm the potential associations between crucial miRNAs and clinical characteristics, including age, tumor stage, tumor grade, residual tumor, overall survival (OS) and disease-free survival (DFS).

Raw counts in TCGA were converted to log-counts per million (CPM) values using the CPM function in edgeR (version 3.4; http://www.bioconductor. org/packages/release/bioc/html/edgeR.html) and normalized using the trimmed mean of M-values algorithm. The preprocessed data were transformed into the gene expression matrix using the voom/Limma R-package (version 3.32.5; http://bioconductor. org/packages/release/bioc/html/limma.html), and then the targeted miRNAs were selected.

The OC samples were dichotomously grouped according to their clinical characteristics (age, $\geq 60$ vs. $<60$; stage, I-II vs. stage III-IV; histologic tumor grade, 1-2 vs. 3-4; with 


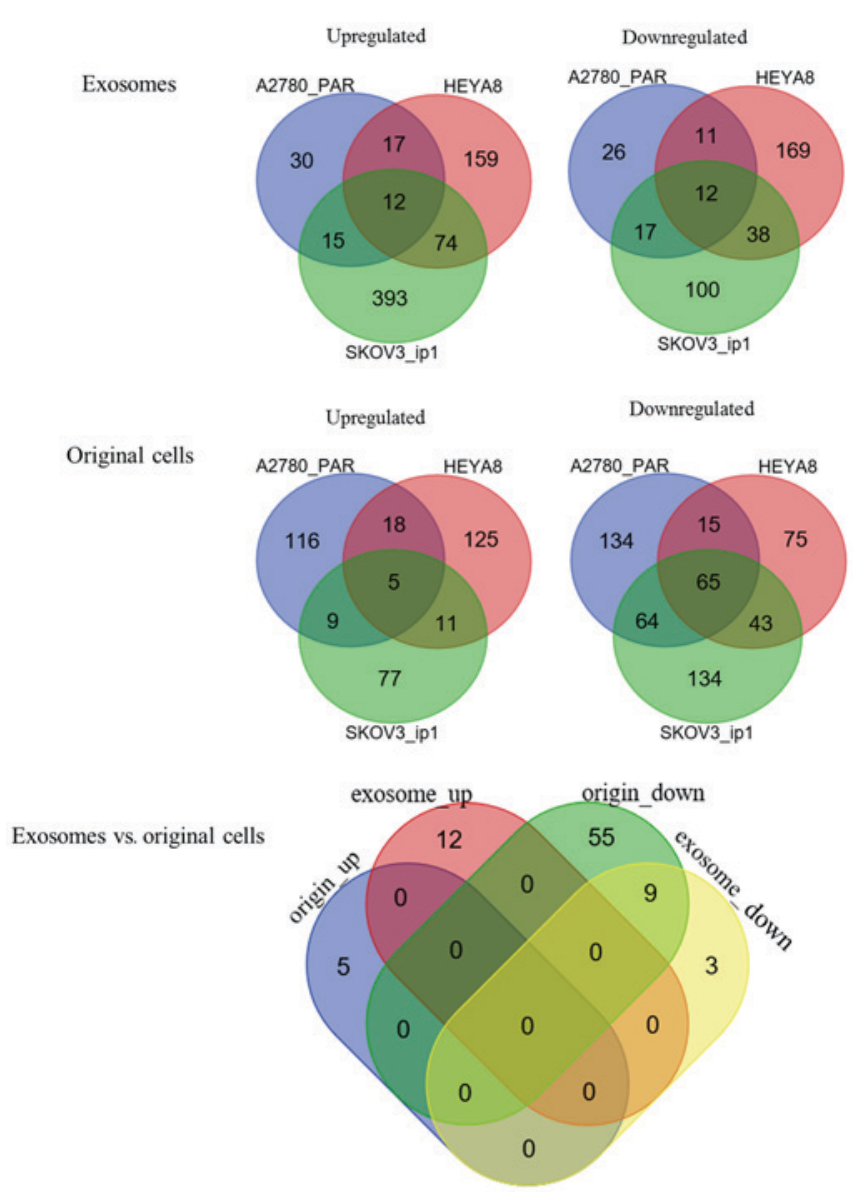

Figure 1. Venn diagram analyzing the common differentially-expressed microRNAs screened in 3 ovarian cancer SKOV3_ip1, A2780_PAR, HeyA8 cell lines and their exosomes when compared with the normal HIO180 cell line, and the difference between ovarian cancer cells and their exosomes.

tumor vs. tumor-free) and the differences in the expression of the aforementioned miRNAs between the two groups were analyzed by an independent t-test. OC samples were staged or graded according to the International Federation of Gynecology and Obstetrics (FIGO) staging (17) or a universal histological grading system (18). The samples with survival data were assigned to two groups based on the expression of each miRNA (low or high expression level). The threshold for the expression group was the median miRNA expression in each samples (high-expression group $\geq$ median; low-expression group $<$ median). The Kaplan-Meier method with the log-rank test was performed using GraphPad Prism software (version 5; GraphPad Software, Inc., La Jolla, CA, USA) to estimate the difference in DFS and OS between the high and low expression groups. $\mathrm{P}<0.05$ was considered to indicate a statistically significant difference.

Furthermore, the clinical associations of miRNAs and mRNA, and the association between miRNA and target genes, were also examined by searching the LinkedOmics database (http://www.linkedomics.org/), which contains multi-omics data and clinical data for 32 cancer types and a total of 11,158 patients from the TCGA project (19). In the LinkedOmics database, the clinical associations of miRNAs and mRNAs, and the association between miRNAs and target genes was directly calculated using Cox's Regression test and Pearson's correlation test, respectively (19).

\section{Results}

Identification of DE-miRNAs between OC and normal control cells. For the exosomal samples, a total of 661 DE-miRNAs were identified between SKOV3_ip1 and HIO180 cells, according to the threshold of $\mathrm{P}<0.05$ and $\log \mathrm{FCl}>1$, including 494 upregulated and 167 downregulated; 141 DE-miRNAs were identified between A2780 _PAR and HIO180 cells, including 74 upregulated and 66 downregulated; and 492 DE-miRNAs were identified between HEYA8 and HIO180 cells, including 262 upregulated and 230 downregulated. These data indicated different profiles of exosomal miRNAs in different cancer cell lines compared with the normal controls (10). To investigate the underlying mechanisms in the development of OC, common miRNAs among the 3 OC cell lines (SKOV3_ip1, A2780 _PAR, and HEYA8, selected as they were not influenced by chemotherapy) were screened by a Venn diagram analysis (Fig. 1). As a result, 12 upregulated and 12 downregulated DE-miRNAs were respectively identified to be shared by the exosomes of these 3 OC cell lines (Table I).

From the original cell samples, a total of 408 DE-miRNAs were identified between SKOV3_ip1 and HIO180 cell lines, including 102 upregulated and 306 downregulated; 426 DE-miRNAs were identified between A2780 _PAR and HIO180 cell lines, including 148 upregulated and 278 downregulated; and 357 DE-miRNAs were identified between HEYA8 and HIO180 cell lines, including 159 upregulated and 198 downregulated. Following the Venn diagram analysis (Fig. 1), 5 upregulated and 65 downregulated DE-miRNAs were identified to be common among the $3 \mathrm{OC}$ cell lines (Table II).

In addition, the number of upregulated genes was increased compared with the number of downregulated genes in the exosomes in each cell line, but the opposite was identified in their original cells, suggesting an overexpression of oncogenes and a decreased expression of tumor-suppressor genes may be the primary mechanisms for exosomal and original cells, respectively, to promote the development and progression of OC. This appeared to be consistent with the established theory that exosomes may transfer certain oncogenic DE-miRNAs into the extracellular environment to maintain and promote tumorigenesis (9). To additionally investigate the differences or similarities in DE-miRNAs between exosomes and their original cells during cancer development, a Venn diagram analysis was performed. The results demonstrated that only 9 downregulated DE-miRNAs were shared between the exosomes and original cells (Fig. 1; Table III), and the others were exosomal-( $n=15)$ or original cell-specific $(n=61)$. These data suggested that these 9 DE-miRNAs may be particularly crucial for OC development in an exosomal or non-exosomal manner.

Target genes for DE-miRNAs between OC cells and normal controls. Following the use of 6 algorithms, only the target genes for 1 upregulated (hsa-miR-940) and 4 shared downregulated (hsa-miR-127-3p, hsa-miR-339-5p, hsa-miR-409-3p and hsa-miR-654-3p) DE-miRNAs of exosomes from 3 OC cells were obtained. From the original cells of the 3 OC cell lines, only the target genes for 1 upregulated (hsa-miR-17-5p) and 19 shared downregulated (hsa-miR-127-3p, hsa-miR-127-5p, 
Table I. Shared DE-microRNAs of exosomes from 3 ovarian cancer A2780_PAR, HEYA8 and SKOV3_ip1 cell lines and the normal ovarian HIO180 cell line.

\begin{tabular}{|c|c|c|c|c|c|c|c|}
\hline \multirow[b]{2}{*}{ Expression } & \multirow[b]{2}{*}{ Symbol } & \multicolumn{2}{|c|}{ SKOV3_ip1 vs. HIO180 } & \multicolumn{2}{|c|}{ A2780_PAR vs. HIO180 } & \multicolumn{2}{|c|}{ HEYA8 vs. HIO180 } \\
\hline & & $\log \mathrm{FC}$ & P-value & $\log \mathrm{FC}$ & P-value & $\operatorname{LogFC}$ & P-value \\
\hline \multirow[t]{12}{*}{ Upregulated } & hsa-miR-6503-3p & 1.5452 & 0.0318 & 4.2537 & 0.0107 & 2.2433 & 0.0018 \\
\hline & hsa-miR-6732-5p & 2.2215 & 0.0052 & 2.2282 & 0.0467 & 1.5404 & 0.0248 \\
\hline & hsa-miR-1825 & 4.3538 & 0.0001 & 4.6936 & 0.0037 & 3.7560 & $3.72 \times 10^{-6}$ \\
\hline & hsa-miR-1281 & 3.7577 & 0.0003 & 3.8823 & 0.0033 & 3.0166 & $8.30 \times 10^{-5}$ \\
\hline & hsa-miR-345-3p & 2.7003 & 0.0016 & 2.1740 & 0.0259 & 2.2642 & 0.0017 \\
\hline & hsa-miR-6865-3p & 1.8265 & 0.0112 & 4.5809 & 0.0069 & 2.0420 & 0.0042 \\
\hline & hsa-miR-3613-5p & 1.7880 & 0.0142 & 3.7595 & 0.0232 & 2.5406 & 0.0006 \\
\hline & hsa-miR-940 & 3.3506 & 0.0005 & 4.6717 & 0.0095 & 3.2752 & $2.71 \times 10^{-5}$ \\
\hline & hsa-let-7f-1-3p & 2.3675 & 0.0032 & 4.2317 & 0.0081 & 2.8521 & 0.0008 \\
\hline & hsa-miR-3921 & 1.2914 & 0.0452 & 4.6335 & 0.0102 & 2.7964 & 0.0002 \\
\hline & hsa-miR-6877-3p & 1.4481 & 0.0326 & 4.1303 & 0.0205 & 1.7630 & 0.0112 \\
\hline & hsa-miR-214-3p & 3.3959 & 0.0005 & 7.3116 & 0.0001 & 1.5850 & 0.0235 \\
\hline \multirow[t]{12}{*}{ Downregulated } & hsa-miR-127-3p & -3.2732 & 0.0006 & -3.8741 & 0.0024 & -2.7713 & 0.0002 \\
\hline & hsa-miR-654-3p & -2.4874 & 0.0087 & -2.7430 & 0.0103 & -2.8694 & 0.0002 \\
\hline & hsa-miR-376c-3p & -2.0892 & 0.0059 & -1.8348 & 0.0462 & -2.6451 & 0.0006 \\
\hline & hsa-miR-487b-3p & -2.0324 & 0.0069 & -3.0766 & 0.0073 & -3.1350 & $6.73 \times 10^{-5}$ \\
\hline & hsa-miR-224-5p & -3.1803 & 0.0008 & -2.5920 & 0.0131 & -4.1790 & $1.06 \times 10^{-6}$ \\
\hline & hsa-miR-6074 & -1.9951 & 0.0332 & -2.1982 & 0.0303 & -2.4949 & 0.0007 \\
\hline & hsa-miR-339-5p & -4.6015 & $7.85 \times 10^{-5}$ & -2.2480 & 0.0377 & -3.3341 & $2.03 \times 10^{-5}$ \\
\hline & hsa-miR-409-3p & -3.5353 & 0.0005 & -2.4868 & 0.0176 & -2.5549 & 0.0006 \\
\hline & hsa-miR-22-3p & -3.1810 & 0.0008 & -5.8766 & 0.0135 & -1.9292 & 0.0061 \\
\hline & hsa-miR-152-3p & -4.0552 & 0.0002 & -3.8035 & 0.0117 & -2.2327 & 0.0019 \\
\hline & hsa-miR-193a-3p & -2.5431 & 0.0026 & -2.9552 & 0.0075 & -2.3664 & 0.0021 \\
\hline & hsa-miR-381-3p & -2.6255 & 0.00195 & -2.2307 & 0.0234 & -2.0432 & 0.0040 \\
\hline
\end{tabular}

DE-miRNAs, differentiability expressed microRNAs; FC, fold change; hsa, Homo sapiens; miR, microRNA.

hsa-miR-140-3p, hsa-miR-140-5p, hsa-miR-184, hsa-miR-299-3p, hsa-miR-299-5p, hsa-miR-337-5p, hsa-miR-339-5p, hsa-miR-409-3p, hsa-miR-409-5p, hsa-miR-485-5p, hsa-miR-543, hsa-miR-574-3p, hsa-miR-604, hsa-miR-654-3p, hsa-miR-654-5p, hsa-miR-767-5p and hsa-miR-935) DE-miRNAs were predicted. Among them, downregulated hsa-miR-127-3p, hsa-miR-339-5p, hsa-miR-409-3p and hsa-miR-654-3p were common between exosomes and their original cells. The regulatory network of these common miRNAs and their target genes is presented in Fig. 2.

Functional enrichment analysis of common DE-miRNAs in exosomes and their original cells. The target genes of the 4 shared downregulated DE-miRNAs between exosomes and their corresponding original cells were analyzed using DAVID for functional enrichment analysis. It was revealed that 6 KEGG pathways were enriched, including the Wnt signaling pathway [hsa-miR-409-3p-Wnt family member 7A (WNT7A), hsa-miR-339-5p-Wnt family member 3A (WNT3A), hsa-miR-654-3p-VANGL planar cell polarity protein 2 (VANGL2), hsa-miR-409-3p-C-terminal binding protein 1 (CTBP1) and hsa-miR-339-5p-chromodomain helicase
DNA-binding protein 8 (CHD8)], Proteoglycans in cancer [hsa-miR-127-3p- serine/threonine-protein phosphatase PP1 $\alpha$ catalytic subunit (PPP1CA), hsa-miR-127-3p-urokinase (PLAU)], and Insulin resistance (hsa-miR-654-3p-ribosomal protein S6 kinase $\alpha 1$; Table IV).

Validation of the association between crucial miRNAs, target genes and clinical characteristics. To validate the clinical importance of the identified miRNAs of the present study, the miRNA expression and clinical data associated with OC were also extracted from the TCGA database. As a result, miRNA expression data were available for 489 patients, but clinical data for age, tumor stage, tumor grade, residual tumor, OS and DFS were obtained from only 481, 281, 472, 431, 485 and 410 women, respectively. The results indicated that the expression of hsa-miR-127 in patients with higher-grade tumors (G3-G4) was significantly decreased compared with that in patients with lower grades (G1-G2) $(11.06 \pm 1.09$ vs. 10.71 \pm 1.53 ; $\mathrm{P}=0.03$; Fig. 3). However, no significant difference in miRNA expression was observed when patients were stratified by age, tumor stage and presence of residual tumors (Table V). Kaplan-Meier analysis also indicated that these miRNAs were not suitable for use as prognostic factors for survival outcomes 
Table II. Shared DE-miRNAs of original cells from 3 ovarian cancer A2780_PAR, HEYA8, SKOV3_ip1 cell lines and the normal ovarian HIO180 cell line. The top 20 downregulated miRNAs were listed.

\begin{tabular}{|c|c|c|c|c|c|c|c|}
\hline \multirow[b]{2}{*}{ Expression } & \multirow[b]{2}{*}{ Symbol } & \multicolumn{2}{|c|}{ SKOV3_ip1 vs. HIO180 } & \multicolumn{2}{|c|}{ A2780_PAR vs. HIO180 } & \multicolumn{2}{|c|}{ HEYA8 vs. HIO180 } \\
\hline & & $\operatorname{LogFC}$ & P-value & LogFC & P-value & $\operatorname{LogFC}$ & P-value \\
\hline \multirow[t]{5}{*}{ Upregulated } & hsa-miR-17-5p & 2.2198 & 0.0008 & 1.8453 & 0.0020 & 1.2775 & 0.0163 \\
\hline & hsa-miR-8057 & 1.3228 & 0.0116 & 1.0111 & 0.0382 & 1.1279 & 0.0340 \\
\hline & hsa-miR-20b-5p & 3.7411 & $2.93 \times 10^{-5}$ & 2.5826 & 0.0002 & 1.2728 & 0.0167 \\
\hline & hsa-miR-20a-5p & 2.3927 & 0.0005 & 1.9455 & 0.0011 & 1.3961 & 0.0087 \\
\hline & hsa-miR-106a-5p & 2.2889 & 0.0006 & 1.7874 & 0.0019 & 1.2947 & 0.0149 \\
\hline \multirow[t]{20}{*}{ Downregulated } & hsa-miR-140-5p & -1.1008 & 0.0319 & -1.4803 & 0.0074 & -2.8567 & $8.16 \times 10^{-8}$ \\
\hline & hsa-miR-487a-3p & -1.7013 & 0.0040 & -1.6719 & 0.0050 & -2.0394 & 0.0001 \\
\hline & hsa-miR-381-3p & -1.7169 & 0.0062 & -1.8955 & 0.0088 & -2.2111 & $3.27 \times 10^{-5}$ \\
\hline & hsa-miR-377-5p & -1.8697 & 0.0158 & -1.0028 & 0.0477 & -1.3828 & 0.0093 \\
\hline & hsa-miR-1306-5p & -2.0760 & 0.0020 & -1.8761 & 0.0019 & -1.7115 & 0.0013 \\
\hline & hsa-miR-370-5p & -1.5049 & 0.0180 & -2.0784 & 0.0010 & -1.5561 & 0.0034 \\
\hline & hsa-miR-6839-5p & -1.1913 & 0.0186 & -1.0604 & 0.0473 & -1.1274 & 0.0340 \\
\hline & hsa-miR-337-5p & -2.1739 & 0.0024 & -2.5794 & 0.0024 & -2.4266 & $5.17 \times 10^{-6}$ \\
\hline & hsa-miR-604 & -1.1959 & 0.0249 & -1.6227 & 0.0032 & -1.049 & 0.0486 \\
\hline & hsa-miR-493-3p & -2.7845 & 0.0002 & -2.5499 & 0.0038 & -3.3224 & $4.54 \times 10^{-10}$ \\
\hline & hsa-miR-758-3p & -2.2183 & 0.0039 & -2.0373 & 0.0008 & -1.8360 & 0.0006 \\
\hline & hsa-miR-127-5p & -2.5959 & 0.0028 & -2.3487 & 0.0015 & -1.9215 & 0.0003 \\
\hline & hsa-mir-381 & -1.5770 & 0.0089 & -1.7904 & 0.0045 & -1.6309 & 0.0022 \\
\hline & hsa-miR-6508-5p & -1.1225 & 0.0339 & -1.0228 & 0.0309 & -1.2049 & 0.0235 \\
\hline & hsa-miR-134-5p & -4.5744 & $2.96 \times 10^{-5}$ & -3.7451 & $1.36 \times 10^{-5}$ & -4.2165 & $2.76 \times 10^{-15}$ \\
\hline & hsa-miR-487b-3p & -5.1189 & $4.09 \times 10^{-6}$ & -5.5027 & $8.78 \times 10^{-7}$ & -5.3287 & $2.17 \times 10^{-23}$ \\
\hline & hsa-miR-323a-3p & -1.4376 & 0.0090 & -1.1565 & 0.0203 & -1.8147 & 0.0006 \\
\hline & hsa-miR-411-3p & -1.9974 & 0.0022 & -1.6534 & 0.0029 & -1.4233 & 0.0075 \\
\hline & hsa-miR-196b-5p & -3.2357 & 0.0017 & -2.2388 & 0.0005 & -3.5772 & $1.95 \times 10^{-11}$ \\
\hline & hsa-miR-370-3p & -1.6817 & 0.0223 & -1.3440 & 0.0090 & -2.1430 & $5.66 \times 10^{-5}$ \\
\hline
\end{tabular}

DE-miRNAs, differentiability expressed microRNAs; FC, fold change; hsa, Homo sapiens; miR, microRNA.

in patients with OC (Fig. 4), which was also confirmed by LinkedOmics analysis using 453 overlapping samples from the miRNASeq and clinical datasets (hsa-miR-409-3p, $\mathrm{P}=0.296$; hsa-miR-339-5p, $\mathrm{P}=0.426$; hsa-miR-127-3p, $\mathrm{P}=0.122$; has-miR-654, $\mathrm{P}=0.071$ ).

A Pearson correlation test using 287 overlapping samples from the miRNASeq and RNAseq datasets validated the significant negative correlation between hsa-miR-409-3p and CTBP1 ( $\mathrm{r}=-0.150, \mathrm{P}=0.011)$, hsa-miR-339-5p and CHD8 $(\mathrm{r}=-0.125, \mathrm{P}=0.033)$ and hsa-miR-127-3p and PPP1CA $(r=-0.201, P=0.0006)$, but not the others. These target genes were also not significantly associated with survival outcomes (CTBP1, $\mathrm{P}=0.966$; CHD8, $\mathrm{P}=0.224$; PPP1CA, $\mathrm{P}=0.333$ ), as demonstrated by a cox regression test using 303 overlapping samples from the RNAseq and clinical datasets.

\section{Discussion}

Although all 3 DE-miRNAs (hsa-miR-127-3p, hsa-miR-339-5p and hsa-miR-409-3p) have been demonstrated to have a tumor-suppressive role in cancer (20), only miR-127-3p (21) and miR-339-5p (22) had been identified in OC cells in previous studies. miR-127-3p was demonstrated to be downregulated in OC cell lines and OC tumor tissues; lentiviral-mediated miR-127-3p reduced the proliferation and invasion of OVCAR-3 and Caov-3 cells by directly inhibiting the Bcl-associated athanogene 5 (BAG5) gene, and subsequent BAG5 upregulation ameliorated the tumor-suppressive effects of miR-127-3p overexpression in OC (21). Although downregulated compared with normal cells, the expression of miR-339-5p was identified to be differentially expressed in different cell types, with the highest expression in OVCAR5 cells and lowest expression in SKOV3 cells (21). Inhibition of miR-339-5p expression increased the migration and invasion of OVCAR5 cells, while upregulated miR-339-5p attenuated this effect in SKOV3 cells (22). The validated targets of miR-339-5p were nucleus accumbens associated 1 and B-cell lymphoma-6 (22). In accordance with these aforementioned studies, hsa-miR-127-3p, hsa-miR-339-5p and hsa-miR-409-3p were also identified to be downregulated in the 3 OC cell lines in the present study. Among them, hsa-miR-127-3p may be particularly important in OC due to its significantly negative correlations with high tumor grades, which were identified in the present study, a key malignant characteristic of cancer. 
Table III. Shared DE-microRNAs between exosomes and original cells.

\begin{tabular}{|c|c|c|c|c|c|c|c|}
\hline \multirow[b]{2}{*}{ Source } & \multirow[b]{2}{*}{ Symbol } & \multicolumn{2}{|c|}{ SKOV3_ip1 vs. HIO180 } & \multicolumn{2}{|c|}{ A2780_PAR vs. HIO180 } & \multicolumn{2}{|c|}{ HEYA8 vs. HIO180 } \\
\hline & & $\operatorname{LogFC}$ & P-value & $\operatorname{LogFC}$ & P-value & $\operatorname{LogFC}$ & P-value \\
\hline \multirow[t]{9}{*}{ Exosomes } & hsa-miR-127-3p & -3.2732 & 0.0006 & -3.8741 & 0.0024 & -2.7712 & 0.0002 \\
\hline & hsa-miR-654-3p & -2.4874 & 0.0087 & -2.7430 & 0.0103 & -2.8694 & 0.0002 \\
\hline & hsa-miR-376c-3p & -2.0893 & 0.0059 & -1.8348 & 0.0462 & -2.6451 & 0.0006 \\
\hline & hsa-miR-487b-3p & -2.0324 & 0.0069 & -3.0766 & 0.0073 & -3.1350 & $6.73 \times 10^{-5}$ \\
\hline & hsa-miR-224-5p & -3.1803 & 0.0008 & -2.5920 & 0.0131 & -4.1790 & $1.06 \times 10^{-6}$ \\
\hline & hsa-miR-339-5p & -4.6015 & $7.85 \times 10^{-5}$ & -2.2480 & 0.0377 & -3.3341 & $2.03 \times 10^{-5}$ \\
\hline & hsa-miR-409-3p & -3.5353 & 0.0005 & -2.4868 & 0.0176 & -2.5549 & 0.0006 \\
\hline & hsa-miR-152-3p & -4.0552 & 0.0002 & -3.8035 & 0.0117 & -2.2327 & 0.0019 \\
\hline & hsa-miR-381-3p & -2.6255 & 0.0020 & -2.2307 & 0.0234 & -2.0432 & 0.0040 \\
\hline \multirow[t]{9}{*}{ Original } & hsa-miR-127-3p & -6.8163 & $5.78 \times 10^{-37}$ & -6.5228 & $1.42 \times 10^{-6}$ & -7.0302 & $3.31 \times 10^{-7}$ \\
\hline & hsa-miR-654-3p & -3.4292 & $1.25 \times 10^{-10}$ & -3.1935 & 0.0006 & -4.0444 & $6.68 \times 10^{-5}$ \\
\hline & hsa-miR-376c-3p & -2.6295 & $7.89 \times 10^{-7}$ & -2.0037 & 0.0062 & -1.6068 & 0.0039 \\
\hline & hsa-miR-487b-3p & -5.3287 & $2.17 \times 10^{-23}$ & -5.1189 & $4.09 \times 10^{-6}$ & -5.5027 & $8.78 \times 10^{-7}$ \\
\hline & hsa-miR-224-5p & -2.6302 & $7.84 \times 10^{-7}$ & -5.7820 & $1.89 \times 10^{-6}$ & -4.4687 & $8.52 \times 10^{-6}$ \\
\hline & hsa-miR-339-5p & -2.0021 & 0.0002 & -1.2081 & 0.0175 & -1.3532 & 0.0118 \\
\hline & hsa-miR-409-3p & -6.0879 & $6.24 \times 10^{-30}$ & -6.4526 & $7.75 \times 10^{-7}$ & -5.2779 & $3.65 \times 10^{-6}$ \\
\hline & hsa-miR-152-3p & -4.7885 & $3.13 \times 10^{-19}$ & -2.8237 & 0.0003 & -2.8109 & 0.0002 \\
\hline & hsa-miR-381-3p & -2.2111 & $3.27 \times 10^{-05}$ & -1.7169 & 0.0062 & -1.8955 & 0.0088 \\
\hline
\end{tabular}

DE-miRNAs, differentiability expressed microRNAs; FC, fold change; hsa, Homo sapiens; miR, microRNA.

Table IV. Function enrichment for target genes of 4 common differentially-expressed microRNAs in exosomes and their original cells of ovarian cancer.

\begin{tabular}{|c|c|c|c|}
\hline KEGG IDs & Pathway name & P-value & Genes \\
\hline hsa00430 & Taurine and hypotaurine metabolism & 0.0068 & GAD2, BAAT, GGT1, GAD1 \\
\hline hsa05205 & Proteoglycans in cancer & 0.0077 & $\begin{array}{l}\text { CAV1, TNF, ERBB4, MRAS, PPP1R12B, MMP9, } \\
\text { WNT3A, PPP1R12C, PLAUR, AKT1, PPP1CA, } \\
\text { CDKN1A, HPSE2, CD44, WNT7A, PLAU }\end{array}$ \\
\hline hsa04310 & Wnt signaling pathway & 0.0139 & $\begin{array}{l}\text { CTNNBIP1, CHD8, CTBP1, WNT3A, VANGL2, } \\
\text { WIF1, BAMBI, DAAM1, TBL1X, WNT7A, TCF7L2, } \\
\text { TCF7L1 }\end{array}$ \\
\hline hsa05412 & $\begin{array}{l}\text { Arrhythmogenic right ventricular } \\
\text { cardiomyopathy }\end{array}$ & 0.0162 & $\begin{array}{l}\text { ITGA9, ACTN4, ITGA7, SGCD, CACNB4, TCF7L2, } \\
\text { TCF7L1, CTNNA2 }\end{array}$ \\
\hline hsa04931 & Insulin resistance & 0.0483 & $\begin{array}{l}\text { AKT1, PRKCZ, PPP1CA, TNF, PTPRF, RPS6KA1, } \\
\text { CREB5, SLC27A2, SLC27A4 }\end{array}$ \\
\hline hsa04520 & Adherens junction & 0.0485 & $\begin{array}{l}\text { PTPRF, ACTN4, SORBS1, CTNND1, TCF7L2, } \\
\text { TCF7L1, CTNNA2 }\end{array}$ \\
\hline
\end{tabular}

KEGG, Kyoto encyclopedia of genes and genomes; hsa, Homo sapiens.

Previous evidence has indicated that the Wnt signaling pathway is crucial for cancer progression (23). Yoshioka et al (24) identified abundant WNT7A in the epithelium of serous $\mathrm{OC}$, but not in borderline and benign tumors, normal ovary or endometrioid carcinomas (24). In vitro analysis revealed that WNT7A may promote the malignant transformation of ovarian epithelial cells by stimulating the $\beta$-catenin/T-cell factor (TCF)-matrix metalloproteinase 7 (MMP7) signaling pathway (24). Stimulation of primary ovarian surface epithelium cells (OSE) in vitro with WNT3A also inhibited glycogen synthase kinase-3 $\beta$ and destabilized the adherens junction through the downregulation of epithelial (E)-cadherin and nuclear translocation of $\beta$-catenin, promoting the increased proliferative rate of OSE 


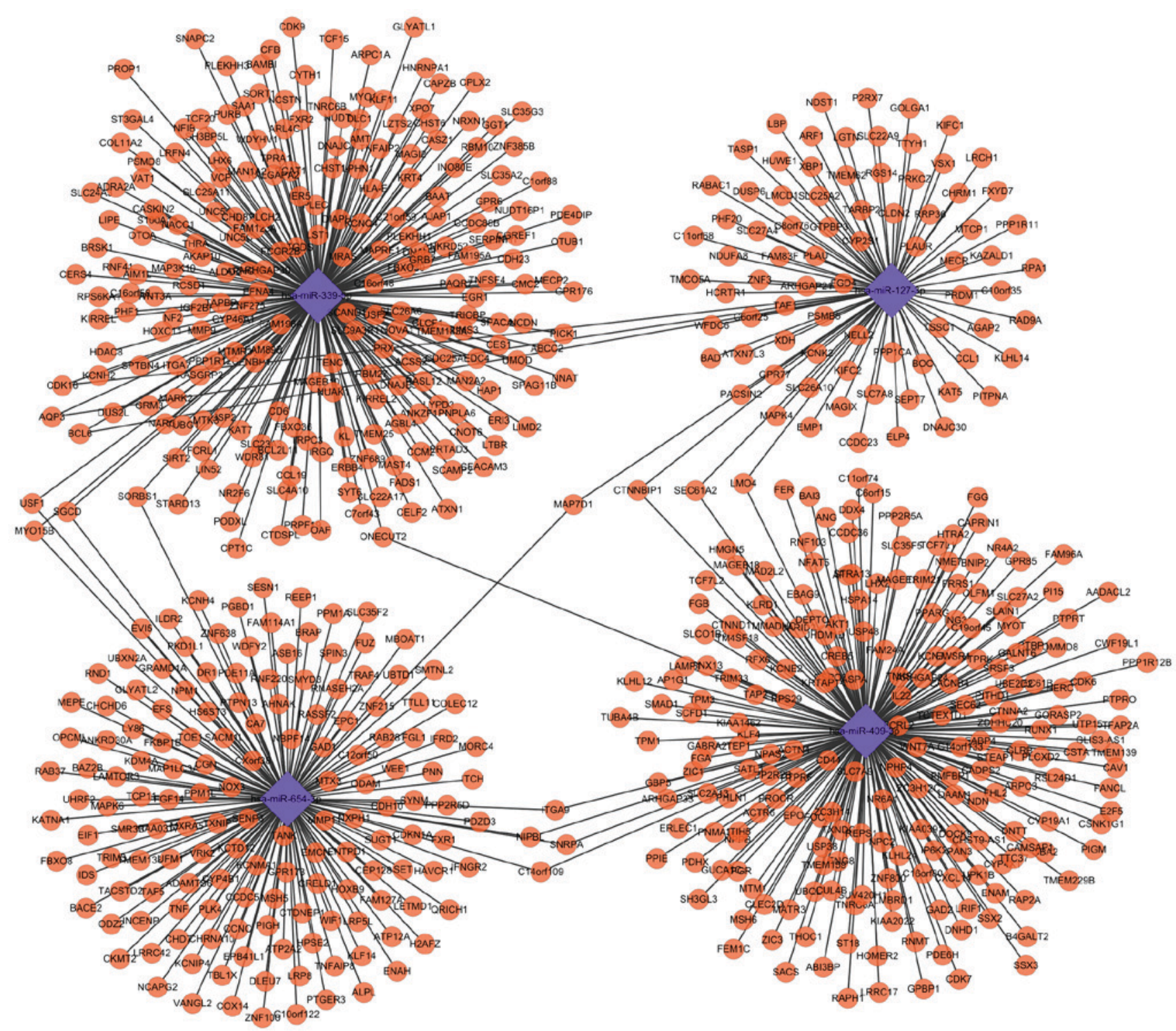

Figure 2. Regulatory network of common differentially-expressed miRNAs in ovarian cancer cells, their exosomes and their target genes. miRNA/miR, microRNA; hsa, Homo sapiens.

cells (25). The archetypal Wnt/planar cell polarity protein VANGL2 has been demonstrated to be overexpressed in basal breast cancer and implicated in tumor growth by activating the VANGL2-nucleoporin p62/sequestosome 1-c-Jun N-terminal kinase pathway, ultimately inducing a poor prognosis (26). CTBP1 was demonstrated to activate the expression of Wnt genes and downregulate their downstream E-cadherin in a TCF-independent manner (27). Small interfering RNA knockdown of CTBP1 restored E-cadherin expression in cancer cell lines, inhibiting their proliferation and invasion (28). CHD8 was previously demonstrated to inhibit the transcription of $\beta$-catenin target genes through chromatin compaction (29) and it may be a tumor suppressor gene (30). However, multiple previous studies have identified an increased expression of CHD8 in cancer cells compared with that in normal tissues $(31,32)$. Subsequent in vitro and in vivo analysis confirmed that CHD8 depletion may be detrimental to the growth of B-acute lymphoblastic leukemia cells and promote its apoptosis (33). The upregulation of Wnt pathway genes in cancer may be due to the downregulation of their corresponding

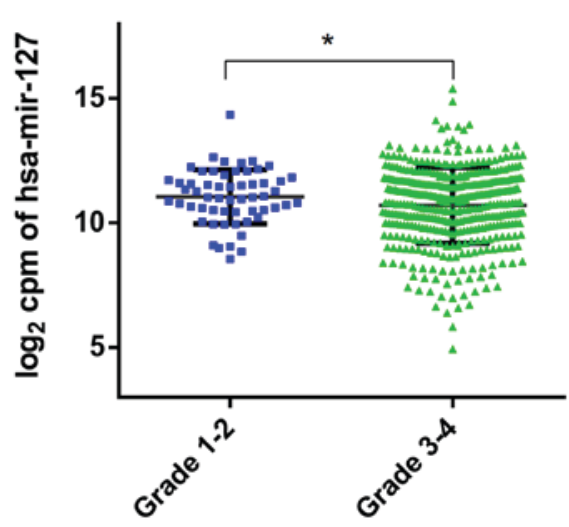

Figure 3. Tumor grade grouping based on miR-127 expression. ${ }^{*} \mathrm{P}<0.05$. miRNA/miR, microRNA; hsa, Homo sapiens; cpm, counts per million.

miRNAs, which was confirmed previously: WNT7A has been demonstrated to be a direct target of miR-15b in OC using a luciferase reporter assay. The inverse correlation of a high 
Table V. Associations between miRNAs and clinical characteristics analyzed using data from The Cancer Genome Atlas data.

\begin{tabular}{|c|c|c|c|c|c|c|c|c|}
\hline Characteristics & hsa-miR-127 & P-value & hsa-miR-339 & P-value & hsa-miR-409 & P-value & hsa-miR-654 & P-value \\
\hline \multicolumn{9}{|l|}{ Age } \\
\hline$\geq 60(n=28)$ & $10.74 \pm 1.49$ & 0.97 & $6.88 \pm 0.92$ & 0.63 & $5.31 \pm 1.40$ & 0.82 & $4.73 \pm 1.47$ & 0.92 \\
\hline$<60(\mathrm{n}=453)$ & $10.75 \pm 1.46$ & & $6.83 \pm 0.98$ & & $5.29 \pm 1.32$ & & $4.74 \pm 1.49$ & \\
\hline \multicolumn{9}{|l|}{ FIGO stage ${ }^{\mathrm{a}}$} \\
\hline I-II (n=28) & $10.10 \pm 1.98$ & 0.08 & $6.61 \pm 1.32$ & 0.16 & $4.81 \pm 1.43$ & 0.05 & $4.18 \pm 1.79$ & 0.95 \\
\hline III-IV (n=253) & $10.79 \pm 1.43$ & & $6.87 \pm 0.92$ & & $5.33 \pm 1.35$ & & $4.77 \pm 1.46$ & \\
\hline \multicolumn{9}{|l|}{ Histological grade } \\
\hline $1-2(n=57)$ & $11.06 \pm 1.09$ & 0.03 & $6.64 \pm 0.82$ & 0.06 & $5.58 \pm 1.15$ & 0.11 & $4.98 \pm 1.20$ & 0.19 \\
\hline $3-4(n=415)$ & $10.71 \pm 1.53$ & & $6.88 \pm 0.95$ & & $5.26 \pm 1.40$ & & $4.70 \pm 1.53$ & \\
\hline \multicolumn{9}{|l|}{ Residual tumor ${ }^{\mathrm{a}}$} \\
\hline Present $(n=317)$ & $10.80 \pm 1.39$ & 0.27 & $6.85 \pm 0.96$ & 0.65 & $5.35 \pm 1.33$ & 0.45 & $4.81 \pm 1.36$ & 0.26 \\
\hline Absent $(n=114)$ & $10.61 \pm 1.71$ & & $6.80 \pm 1.01$ & & $5.24 \pm 1.42$ & & $4.63 \pm 1.81$ & \\
\hline
\end{tabular}

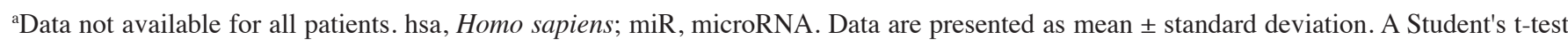
was used for the statistical analysis.

A
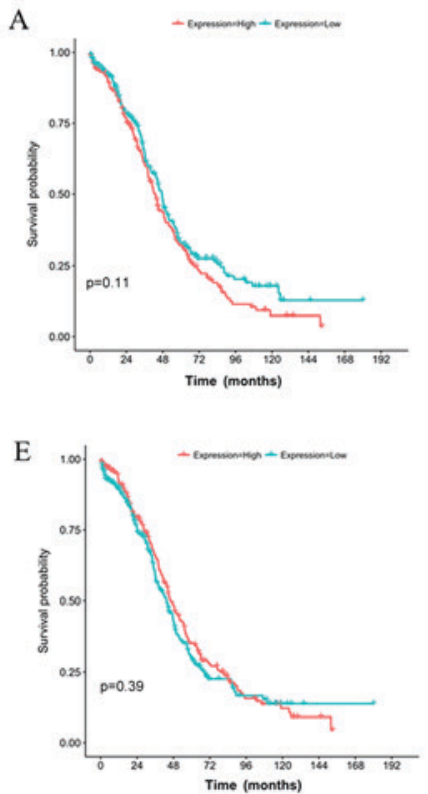

B
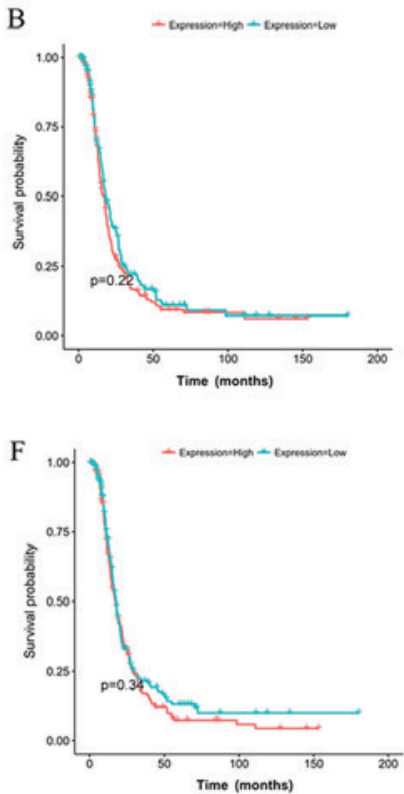

C
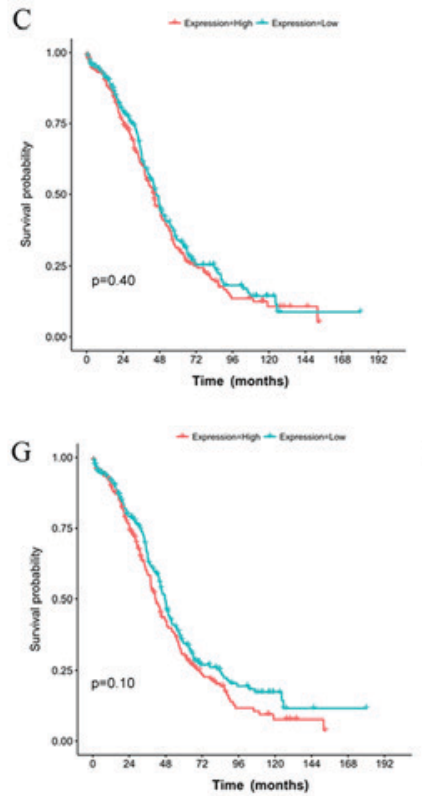

D

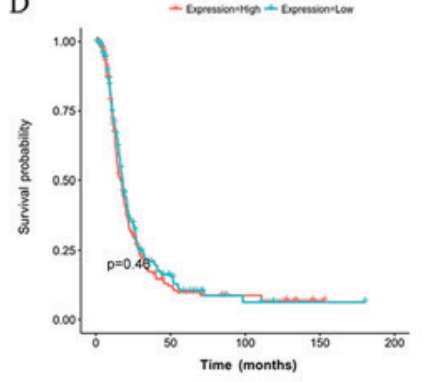

H

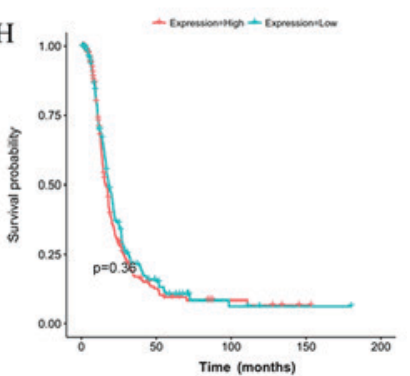

Figure 4. Kaplan-Meier analysis indicating that these miRNAs are not good prognostic factors associated with survival outcomes for patients with ovarian cancer. (A) OS of miR-127; (B) DFS of miR-127; (C) OS of miR-409; (D) DFS of miR-409; (E) OS of miR-339; (F) DFS of miR-339; (G) OS of miR-654; (H) DFS of miR-654. OS, overall survival; DFS, disease free survival; miRNAs, microRNAs.

expression of WNT7A and low-expression of miR-15b in OC was observed to be associated with decreased survival rates in patients with OC (34). miR-137 has been revealed to suppress the 3' UTR luciferase-reporter activity of CTBP1, reducing its mRNA expression levels followed by increasing the expression of immediate downstream effectors of CtBP1, including E-cadherin (35). In the present study, it was identified that WNT7A/CTBP1, WNT3A/CHD8, and VANGL2 were respectively regulated by hsa-miR-409-3p, hsa-miR-339-5p and hsa-miR-654-3p, thereby indirectly explaining the roles of these miRNAs in OC. However, only negative regulatory associations between hsa-miR-409-3p and CTBP1, and hsa-miR-339-5p and CHD8, were verified using the OC data in
TCGA, which had not been demonstrated in previous studies, to the best of our knowledge. These data indicate that these 2 miRNAs may be novel effectors in OC.

PLAU encodes for the urokinase-type plasminogen activator (uPA), which has been demonstrated to be critical for $\mathrm{OC}$ development and progression (36,37). uPA is overexpressed in the majority of patients with OC (36). The overexpression of uPA has been significantly associated with poorer degrees of differentiation, higher clinical stages (III-IV), lymph node or omental metastasis and decreased survival $(36,37)$. Mechanistic studies suggested that uPA may promote the migration and invasion of OC cells by inducing the formation of vasculogenic mimicry via the Protein kinase $\mathrm{B} / \mathrm{mechanistic}$ target 
of rapamycin/MMP2/Laminin5 $\gamma 2$ signaling pathway, while the use of an uPA antagonist, cyclic-arginine-glycine-aspartic acid, weakened these processes (38). In addition, the upregulated expression of uPA in OC cells was demonstrated to be p38 mitogen activated protein kinase (MAPK) signal-pathway dependent: The utility of p38 MAPK inhibitor SB203580 almost decreased the expression of UPA to an absolute count of 0 (39). Furthermore, uPA may be a target gene of miR-193b: Overexpression of miR-193b was demonstrated to negatively regulate uPA expression and thereby inhibit the invasiveness of breast (40), pancreatic (41) and prostate cancer (42) cells. However, to the best of our knowledge, there have been no prior studies investigating uPA-associated miRNAs in OC cells. In the present study, it was predicted that the upregulation of UPA in OC may be attributed to the downregulation of hsa-miR-127-3p. This was not verified by subsequent TCGA analysis, and therefore additional experiments may be required.

PPP1CA, encoding PP1 $\alpha$, is suggested to regulate critical cellular events, including cell cycle and apoptosis $(43,44)$; therefore, it may be a potential tumor-associated gene. This hypothesis has been confirmed by previous studies: For example, Hsu et al (45) identified that PPP1CA was amplified and overexpressed in oral squamous cell carcinomas cells (OSCC), and that knockdown of PP1 $\alpha$ suppressed OSCC cell G0 growth arrest by modulating retinoblastoma phosphorylation. Nohata et al (46) also observed the overexpression of PPP1CA in clinical specimens with maxillary sinus squamous cell carcinoma (MSSCC). Silencing of the PPP1CA gene significantly inhibited cancer cell proliferation and invasion. Shastry et al (47) revealed that patients with glioblastoma with positive PP1A and p53 expression exhibited a poorer overall median time (14 months) compared with PP1A-negative cases (21 months). Accordingly, we hypothesize that PPP1CA is also highly expressed in OC. Furthermore, PP1CA was revealed to be expressed at high levels due to a frequent downregulation of miR-874 in MSSCC (46). However, to the best of our knowledge, no previous studies have investigated PPP1CA-associated miRNAs in OC cells. In the present study, it was predicted that the upregulation of PPP1CA in OC may be attributed to the downregulation of hsa-miR-127-3p. This negative association was also verified in the TCGA analysis.

Notably, it has been suggested that co-culture with OC ES-2 cells and incubation with ES-2-conditioned medium induced the expression of uPA mRNA in fibroblastic stromal LEP cells $\sim 2$-fold (48), indicating that OC cells may externally secret certain signals, including miRNAs, to regulate the expression of uPA and induce tumorigenesis. As expected, hsa-miR-127-3p was also identified to be downregulated in the exosomes of OC cells in the present study, and therefore the addition of hsa-miR-127-3p-containing exosomes may be a novel approach to the treatment of OC. Similarly, Lin et al (49) observed that exosomes derived from the conditional supernatant of the esophageal cancer KYSE410 cell line induced an increase in the cell migratory and invasive abilities of KYSE410 and 2 other esophageal carcinoma KYSE510 and YES2 cell lines, in which the Wnt/ $\beta$-catenin signaling pathway was activated. Accordingly, miRNAs that regulate Wnt pathway genes in exosomes may also be downregulated. This hypothesis may be indirectly supported by data from Dempsey et al (50), who indicated that platelet exosomes were enriched for miRNAs that regulated WNT pathways. By transfecting miRs into endothelial cells, these platelet exosomal miRNAs were able to inhibit WNT signaling in endothelial cells and affect cell motility (50). In the present study, the expressions of hsa-miR-339-5p and hsa-miR-409-3p that regulated Wnt pathways genes were also significantly decreased in the exosomes of OC cells, indicating their potential for OC treatment through overexpression.

There are certain limitations to be acknowledged when interpreting these results. Firstly, only 3 OC cell lines were selected for screening crucial miRNAs. Therefore, the miRNAs identified here may not represent miRNA candidates that are potentially associated with all types of OC. Secondly, only 2 repeats were included in each sample of the microarray data used (12), which may affect the results of the statistical analysis. However, it may be acceptable to perform bioinformatics analyses, as demonstrated by several previous studies $(51,52)$. Thirdly, the TCGA data did not include the normal control, which led to several unexpected, non-significant associations between the miRNAs identified in OC cells and clinical characteristics. Additional clinical trials are required to confirm the conclusions. Fourthly, although the analysis of the present study has preliminarily demonstrated a negative association between miRNAs and their target genes (hsa-miR-409-3p and CTBP1, hsa-miR-339-5p and CHD8, and hsa-miR-127-3p and PPP1CA) in clinical samples, in vitro and in vivo experiments are necessary. Fifthly, the exosomal mechanisms of these 3 miRNAs remain uninvestigated, which require additional confirmation by using an exosome inhibitor, GW4869 (53).

In conclusion, the present study preliminarily revealed that hsa-miR-127-3p, hsa-miR-339-5p and hsa-miR-409-3p may be crucial for the development of $\mathrm{OC}$ in exosomal- and non-exosomal-based pathways. They may be involved in OC by targeted modulation of WNT (CTBP1 and CHD8) or the Proteoglycans in cancer pathway (PPP1CA) genes. Upregulation of these genes may be a potential therapeutic strategy for OC.

\section{Acknowledgements}

Not applicable.

\section{Funding}

No funding was received.

\section{Availability of data and materials}

The datasets generated and/or analyzed during the current study are available in the NCBI database repository (https://www. ncbi.nlm.nih.gov/geo/query/acc.cgi?acc=GSE76449).

\section{Authors' contributions}

SZ and YY participated in the design of this study. XZ and XF performed the statistical and bioinformatics analyses. SZ, WL, SX and YY contributed to the acquisition and interpretation of data. SZ and YY drafted and revised the manuscript. All authors read and approved the final manuscript. 


\section{Ethics approval and consent to participate}

Not applicable.

\section{Consent for publication}

Not applicable.

\section{Competing interests}

The authors declare that they have no competing interests.

\section{References}

1. Chen W, Zheng R, Baade P, Zhang S, Zeng H, Bray F, Jemal A, Yu XQ and He J: Cancer statistics in China, 2015. CA-Cancer J Clin 66: 115-132, 2016

2. Cliby WA, Powell MA, Al-Hammadi N, Chen L, Philip Miller J, Roland PY, Mutch DG and Bristow RE: Ovarian cancer in the United States: Contemporary patterns of care associated with improved survival. Gynecol Oncol 136: 11-17, 2015.

3. Hausser $\mathbf{J}$ and Zavolan M: Identification and consequences of miRNA-target interactions-beyond repression of gene expression. Nat Rev Genet 15: 599-612, 2014.

4. Yang Z, Wang XL, Bai R, Liu WY, Li X, Liu M and Tang H: miR-23a promotes IKK $\alpha$ expression but suppresses ST7L expression to contribute to the malignancy of epithelial ovarian cancer cells. Br J Cancer 115: 731-740, 2016.

5. Shen W, Song M, Liu J, Qiu G, Li T, Hu Y and Liu H: MiR-26a promotes ovarian cancer proliferation and tumorigenesis. PLoS One 9: e86871, 2014.

6. Dong P, Xiong Y, Watari H, Hanley SJ, Konno Y, Ihira K, Yamada T, Kudo M, Yue J and Sakuragi N: MiR-137 and miR-34a directly target Snail and inhibit EMT, invasion and sphere-forming ability of ovarian cancer cells. J Exp Clin Cancer Res 35: 132, 2016.

7. Neviani P and Fabbri M: Exosomic microRNAs in the tumor microenvironment. Front Med (Lausanne) 2: 47, 2015.

8. Baroni S, Romero-Cordoba S, Plantamura I, Dugo M, D'Ippolito E, Cataldo A, Cosentino G, Angeloni V Rossini A, Daidone MG and Iorio MV: Exosome-mediated delivery of miR-9 induces cancer-associated fibroblast-like properties in human breast fibroblasts. Cell Death Dis 7: e2312, 2016.

9. Singh R, Pochampally R, Watabe K, Lu Z and Mo YY: Exosome-mediated transfer of miR-10b promotes cell invasion in breast cancer. Mol Cancer 13: 256, 2014.

10. Kobayashi M, Salomon C, Tapia J, Illanes SE, Mitchell MD and Rice GE: Ovarian cancer cell invasiveness is associated with discordant exosomal sequestration of Let-7 miRNA and miR-200. J Transl Med 12: 4, 2014.

11. Ying $X$, Wu Q, Wu X, Zhu Q, Wang X, Jiang L, Chen $X$ and Wang X: Epithelial ovarian cancer-secreted exosomal miR-222-3p induces polarization of tumor-associated macrophages. Oncotarget 7: 43076-43087, 2016.

12. Kanlikilicer P, Rashed MH, Bayraktar R, Mitra R, Ivan C, Aslan B, Zhang X, Filant J, Silva AM, Rodriguez-Aguayo $\mathrm{C}$, et al: Ubiquitous release of exosomal tumor suppressor miR-6126 from ovarian cancer cells. Cancer Res 76: 7194-7207, 2016

13. De A, Powers B, De A, Zhou J, Sharma S, Van Veldhuizen P, Bansal A, Sharma R and Sharma M: Emblica officinalis extract downregulates pro-angiogenic molecules via upregulation of cellular and exosomal miR-375 in human ovarian cancer cells. Oncotarget 7: 31484-31500, 2016.

14. Smyth GK: Limma: Linear models for microarray data. In: Bioinform Comput Biol Solut Using R Bioconduct. Gentleman R, Carey V, Dudoit S, Irizarry R, Huber W (eds). Springer, New York, NY, pp397-420, 2005.

15. Kohl M, Wiese S and Warscheid B: Cytoscape: Software for visualization and analysis of biological networks. Methods Mol Biol 696: 291-303, 2011

16. Huang da W, Sherman BT and Lempicki RA: Systematic and integrative analysis of large gene lists using DAVID bioinformatics resources. Nat Protoc 4: 44-57, 2009.
17. Kosary CL: FIGO stage, histology, histologic grade, age and race as prognostic factors in determining survival for cancers of the female gynecological system: An analysis of 1973-87 SEER cases of cancers of the endometrium, cervix, ovary, vulva, and vagina. Semin Surg Oncol 10: 31-46, 1994.

18. Shimizu Y, Kamoi S, Amada S, Akiyama F and Silverberg SG: Toward the development of a universal grading system for ovarian epithelial carcinoma: Testing of a proposed system in a series of 461 patients with uniform treatment and follow-up. Cancer 82: 893-901, 1998.

19. Vasaikar SV, Straub P, Wang J and Zhang B: LinkedOmics: Analyzing multi-omics data within and across 32 cancer types. Nucleic Acids Res 46(D1): D956-D963, 2018.

20. Zhang G, Liu Z, Xu H and Yang Q: miR-409-3p suppresses breast cancer cell growth and invasion by targeting Akt1. Biochem Biophys Res Commun 469: 189-195, 2016.

21. Bi L, Yang Q, Yuan J, Miao Q, Duan L, Li F and Wang S: MicroRNA-127-3p acts as a tumor suppressor in epithelial ovarian cancer by regulating the BAG5 gene. Oncol Rep 36: 2563-2570, 2016.

22. Shan W, Li J, Bai Y and Lu X: miR-339-5p inhibits migration and invasion in ovarian cancer cell lines by targeting NACC1 and BCL6. Tumour Biol 37: 5203-5211, 2016.

23. Arend RC, Londoño-Joshi AI, Straughn JM Jr and Buchsbaum DJ: The Wnt/ $\beta$-catenin pathway in ovarian cancer: A review. Gynecol Oncol 131: 772-779, 2013

24. Yoshioka S, King ML, Ran S, Okuda H, MacLean JA II, Mcasey ME, Sugino N, Brard L, Watabe K and Hayashi K: WNT7A regulates tumor growth and progression in ovarian cancer through the WNT/3-catenin pathway. Mol Cancer Res 10: 469-482, 2012.

25. Usongo M, Li X and Farookhi R: Activation of the canonical WNT signaling pathway promotes ovarian surface epithelial proliferation without inducing $\beta$-catenin/Tcf-mediated reporter expression. Dev Dyn 242: 291-300, 2013.

26. Puvirajesinghe TM, Bertucci F, Jain A, Scerbo P, Belotti E, Audebert S, Sebbagh M, Lopez M, Brech A, Finetti P, et al: Identification of p62/SQSTM1 as a component of non-canonical Wnt VANGL2-JNK signalling in breast cancer. Nat Commun 7: 10318, 2016.

27. Deng Y, Deng H, Liu J, Han G, Malkoski S, Liu B, Zhao R, Wang XJ and Zhang Q: Transcriptional down-regulation of Brcal and E-cadherin by CtBP1 in breast cancer. Mol Carcinog 51: 500-507, 2012.

28. Wang R, Asangani IA, Chakravarthi BV, Ateeq B, Lonigro RJ, Cao Q, Mani RS, Camacho DF, McGregor N, Schumann TE, et al: Role of transcriptional corepressor CtBP1 in prostate cancer progression. Neoplasia 14: 905-914, 2012.

29. Nishiyama M, Skoultchi AI and Nakayama KI: Histone H1 recruitment by CHD8 is essential for suppression of the Wnt- $\beta$-catenin signaling pathway. Mol Cell Biol 32: 501-512, 2012.

30. Sawada G, Ueo H, Matsumura T, Uchi R, Ishibashi M, Mima K, Kurashige J, Takahashi Y, Akiyoshi S, Sudo T, et al: CHD8 is an independent prognostic indicator that regulates $\mathrm{Wnt} / \beta$-catenin signaling and the cell cycle in gastric cancer. Oncol Rep 30: $1137-1142,2013$

31. Menon T, Yates JA and Bochar DA: Regulation of androgen-responsive transcription by the chromatin remodeling factor CHD8. Mol Endocrinol 24: 1165-1174, 2010.

32. Jones DH and Lin DI: Amplification of the NSD3-BRD4-CHD8 pathway in pelvic high-grade serous carcinomas of tubo-ovarian and endometrial origin. Mol Clin Oncol 7: 301-307, 2017.

33. Shingleton JR and Hemann MT: The chromatin regulator CHD8 is a context-dependent mediator of cell survival in murine hematopoietic malignancies. PLoS One 10: e0143275, 2015.

34. MacLean JA II, King ML, Okuda $\mathrm{H}$ and Hayashi K: WNT7A regulation by miR-15b in ovarian cancer. PLoS One 11: e0156109, 2016

35. Deng Y, Deng H, Bi F, Liu J, Bemis LT, Norris D, Wang XJ and Zhang Q: MicroRNA-137 targets carboxyl-terminal binding protein 1 in melanoma cell lines. Int J Biol Sci 7: 133-137, 2011.

36. Wang L, Madigan MC, Chen H, Liu F, Patterson KI, Beretov J, O'Brien PM and Li Y: Expression of urokinase plasminogen activator and its receptor in advanced epithelial ovarian cancer patients. Gynecol Oncol 114: 265-272, 2009.

37. Dorn J, Harbeck N, Kates R, Gkazepis A, Scorilas A, Soosaipillai A, Diamandis E, Kiechle M, Schmalfeldt B and Schmitt M: Impact of expression differences of kallikrein-related peptidases and of UPA and PAI-1 between primary tumor and omentum metastasis in advanced ovarian cancer. Ann Oncol 22: $877-883,2011$ 
38. Tang J, Wang J, Fan L, Li X, Liu N, Luo W, Wang J, Wang Y and Wang Y: cRGD inhibits vasculogenic mimicry formation by down-regulating uPA expression and reducing EMT in ovarian cancer. Oncotarget 7: 24050-24062, 2016.

39. Estrella VC, Eder AM, Liu S, Pustilnik TB, Tabassam FH, ClaretFX, Gallick GE, Mills GB and Wiener JR: Lysophosphatidic acid induction of urokinase plasminogen activator secretion requires activation of the p38MAPK pathway. Int J Oncol 31: 441-449, 2007.

40. Li XF, Yan PJ and Shao ZM: Downregulation of miR-193b contributes to enhance urokinase-type plasminogen activator (uPA) expression and tumor progression and invasion in human breast cancer. Oncogene 28: 3937-3948, 2009.

41. Li J, Kong F, Wu K, Song K, He J and Sun W: miR-193b directly targets STMN1 and uPA genes and suppresses tumor growth and metastasis in pancreatic cancer. Mol Med Rep 10: 2613-2620, 2014.

42. Xie C, Jiang XH, Zhang JT, Sun TT, Dong JD, Sanders AJ, Diao RY, Wang Y, Fok KL, Tsang LL, et al: CFTR suppresses tumor progression through miR-193b targeting urokinase plasminogen activator (uPA) in prostate cancer. Oncogene 32 : 2282-2291, 2291.e1-e7, 2013.

43. Kawabe T, Muslin AJ and Korsmeyer SJ: HOX11 interacts with protein phosphatases PP2A and PP1 and disrupts a G2/M cell-cycle checkpoint. Nature 385: 454-458, 1997.

44. Dessauge F, Cayla X, Albar JP, Fleischer A, Ghadiri A, Duhamel $\mathrm{M}$ and Rebollo A: Identification of PP1alpha as a caspase-9 regulator in IL-2 deprivation-induced apoptosis. J Immunol 177: 2441-2451, 2006.

45. Hsu LC, Huang X, Seasholtz S, Potter DM and Gollin SM: Gene amplification and overexpression of protein phosphatase 1 alpha in oral squamous cell carcinoma cell lines. Oncogene 25 : 5517-5526, 2006.

46. Nohata N, Hanazawa T, Kikkawa N, Sakurai D, Fujimura L, Chiyomaru T, Kawakami K, Yoshino H, Enokida $\mathrm{H}$, Nakagawa M, et al: Tumour suppressive microRNA-874 regulates novel cancer networks in maxillary sinus squamous cell carcinoma. Br J Cancer 105: 833-841, 2011.
47. Shastry AH, Thota B, Srividya MR, Arivazhagan A and Santosh V: Nuclear Protein Phosphatase $1 \alpha$ (PP1A) expression is associated with poor prognosis in p53 expressing glioblastomas. Pathol Oncol Res 22: 287-292, 2016.

48. Noskova V, Ahmadi S, Asander E and Casslén B: Ovarian cancer cells stimulate uPA gene expression in fibroblastic stromal cells via multiple paracrine and autocrine mechanisms. Gynecol Oncol 115: 121-126, 2009.

49. Lin F, Wang HJ, Li CX, Li H, Wang T, Nan P, Qian HL and Zhan QM: Effects of esophageal cancer cell-derived exosomes on cancer cell migration and invasion and its mechanism research. Med J Chin PLA 42: 307-313, 2017.

50. Dempsey E, Dervin F and Maguire PB: Platelet derived exosomes are enriched for specific microRNAs which regulate WNT signalling in endothelial cells. Blood 124: 2760, 2014.

51. Zhang P, Garnett J, Creighton CJ, Al Sannaa GA, Igram DR, Lazar A, Liu X, Liu C and Pollock RE: EZH2-miR-30d-KPNB1 pathway regulates malignant peripheral nerve sheath tumour cell survival and tumourigenesis. J Pathol 232: 308-318, 2014.

52. Li B, Jiang S, Yu X, Cheng C, Chen S, Cheng Y, Yuan JS, Jiang D, He P and Shan L: Phosphorylation of trihelix transcriptional repressor ASR3 by MAP KINASE4 negatively regulates Arabidopsis immunity. Plant Cell 27: 839-856, 2015.

53. Rashed MH, Kanlikilicer P, Rodriguez-Aguayo C, Pichler M, Bayraktar R, Bayraktar E, Ivan C, Filant J, Silva A, Aslan B, et al: Exosomal miR-940 maintains SRC-mediated oncogenic activity in cancer cells: A possible role for exosomal disposal of tumor suppressor miRNAs. Oncotarget 8: 20145-20164, 2017.

(i) (2) This work is licensed under a Creative Commons Attribution-NonCommercial-NoDerivatives 4.0 International (CC BY-NC-ND 4.0) License. 